\title{
Advances in Cancer Therapy: Targeted Therapies
}

\section{Dieter Marmé}

Klinik für Tumorbiologie, Freiburg i.Br., Germany

As we learned to understand many of the mechanisms which drive cancer development and progression, a paradigmatic change for new treatment options occurred. Instead of focusing mostly on unspecific cytotoxic therapeutics, new substances directed against cancer-driving molecules made their way to the clinic. The idea behind this concept of 'targeted cancer therapies' is quite simple: if we are able to bring cancer-driving molecules under control, we might be able to stop cancer development and progression. An outstanding example confirming this hypothesis is the successful treatment of chronic myeloid leukemia (CML) with imatinib. This treatment works so perfectly because the underlying genetic alteration of CML is a chromosomal translocation that leads to a constitutive activation of the BCL-Abl tyrosine kinase - imatinib inhibits its activity.

Interestingly, after the successful use of imatinib against CML, it was found that the same compound could also be applied to treat gastrointestinal stromal tumors (GIST). Here again, the explanation is rather simple: imatinib not only inhibits the BCL-Abl-tyrosine kinase (one of about 500 protein kinases coded in the human genome), but also the c-KIT tyrosine kinase, which is mutated and activated in GIST. Furthermore in about $5-7 \%$ of GIST patients, the platelet-derived growth factor receptor alpha (PDGFRA) is mutated and activated. Its activity is also inhibited by imatinib. This explains to some degree the successful use of imatinib in the treatment of GIST.

What does the imatinib story tell us?

(i) 'Targeted therapy' does work effectively (CML); (ii) a targeting compound does not necessarily have to be extremely specific. In the case of GIST, imatinib modulates the activity of 2 tumordriving mutated protein kinases: BCL-Abl tyrosine kinase and PDGFRA tyrosine kinase. (iii) Activated protein kinases are often involved in tumor development and thus are potential targets for treatment.

However, most tumors have a much more complex etiology than CML. Over time, sometimes over decades, they progress by accumulating many mutations, occurring in so-called oncogenes and/or tumor suppressor genes. This process results in progression of the disease and formation of metastases. This process (the types of mutations and their temporal occurrence) differs from tumor type to tumor type. Furthermore, within a given tumor entity, this process also differs from patient to patient.

Thus, at present we are faced with quite a number of problems, which have to be solved in order to proceed with the development of 'targeted therapies':

- Identification of the molecular identities of tumor-driving mechanisms and their validation as 'therapeutic targets'. In this area considerable progress has been made over the last years.

- Diagnostic verification of the presence of therapeutic targets in the tumor of each individual patient. This gives us the necessary information as a prerequisite for 'personalized cancer therapy'. Molecular pathology has successfully developed novel technologies, i.e. molecular and histological methods, such as nextgeneration sequencing or semi-quantitative immuno-histochemistry. A large effort has to be made to integrate these technologies into clinical studies and thus eventually to facilitate more refined guidelines, combining validated diagnostics with precision therapy in clinical practice.

- Development of more compounds suited for targeted therapy. A large number of molecules and antibodies is already available. Many of them have been investigated in clinical studies and some of these have already found their way to the clinic.

The knowledge of the individual transforming entities will help to stratify patients into subgroups suited for specific targeted treatments. This has already been shown to be a successful strategy. However, this approach might require large amounts of tumor tissue (and does not necessarily solve the problem of tumor heterogeneity). Thus alternative sources of tumor samples, such as blood (i.e. liquid biopsies), have to be explored to gain access to the complete signature of an individual tumor. First successful examples encourage us to proceed in this direction.

These activities are ongoing. Together with the availability of more and quantitated target-specific diagnostic assays, targeted therapies will become increasingly successful in treating cancer. As in general tumors are driven by more than one oncogenic process, the combination of two or even more targeted compounds could increase their curative potential. So far, many of these novel treat-

\section{KARGER}

() 2016 S. Karger GmbH, Freiburg

Fax +497614520714 
ment options are applied in combination with traditional cytotoxic substances.

In this issue of ONCOLOGY Research AND TREATMENT experts in the field summarize the achievements reached so far in selected tumor entities. They also address the deficits as they show up at present and give an outlook on the urgent needs in order to accomplish successful targetined therapy.

Lung cancer is a prominent example where substantial progress has been made. Michels and Wolf [1] in particular address resistance problems and also the blood/brain barrier as a major hurdle to treat brain metastases. The therapy of chronic lymphocytic leukemia (CLL) can be improved in certain cases by combining different agents addressing different targets [2]. Head and neck cancer requires substantially more clinical studies investigating new compounds addressing the targets that have been shown to be involved in this disease [3]. To proceed with targeted therapy of esophagogastric cancer, we need much better diagnostics and more information on markers. This should considerably improve the evaluation of clinical investigations [4]. Metastatic colorectal cancer (mCRC) is treated basically by targeting an oncogenic molecule at the surface of the cancer cell on the one hand and tumor angiogenesis as a target coming from the cellular environment on the other [5]. As targeted therapies require substantial support from molecular pathology, Manfred Dietel [6] describes the current situation in this field and addresses the problems and the future developments.

In summary, personalized targeted therapies are at present the most encouraging treatment strategies to advance in the battle against cancer. The first steps in this direction have been successful. However, there is still a long way to go.

\section{Disclosure Statement}

The Author declares no conflicts of interest.

\section{References}

1 Michels S, Wolf J:Targeted therapy of lung cancer. Oncol Res Treat 2016;39: DOI: $10.1159 / 000453406$.

2 Al-Sawaf O, Fischer K, Eichhorst B, Hallek M: Targeted therapy of CLL. Oncol Res Treat 2016;39: DOI: 10.1159/000452786.

3 Rieke DT, Klinghammer K, Keilholz U: Targeted therapy of head and neck cancer. Oncol Res Treat 2016;39: DOI: 10.1159/000452432.

4 Kopp HG, Hofheinz RD: Targeted treatment of esophagogastric cancer. Oncol Res Treat 2016;39: DOI: 10.1159/000452877.

5 Seeber A, Gastl G: Targeted therapy of colorectal cancer. Oncol Res Treat 2016;39: DOI: $10.1159 / 000453027$.

6 Dietel M: Molecular pathology: a requirement for precision medicine in cancer. Oncol Res Treat 2016;39: DOI: 10.1159/000453085. 